\title{
A Case of Seronegative Pulmonary-renal Syndrome: Diagnostic and Therapeutic Challenge
}

Shkelqim Muharremi1*, Aleksandar Poposki ${ }^{1}$, Vangelka Kovaceska ${ }^{1}$, Liljana Tozija ${ }^{2}$, Gordana Petrusevska ${ }^{3}$, Biljana Gerasimovska², Blerim Bexheti², Nikola Gjorgjievski², Zoran Janevski ${ }^{2}$, Muhamed Saidi², Adrijana Spasovska ${ }^{2}$, Bojana Poposka², Gjulsen Selim²

${ }^{1}$ Department of Nephrology, Institute of Nephrology, Struga, Republic of Macedonia; ${ }^{2}$ Department of ICU, University Clinic of Nephrology, Medical Faculty, Ss. Cyril and Methodius University, Skopje, Republic of Macedonia; ${ }^{3}$ Department of Pathology, Ss. Cyril and Methodius University, Skopje, Republic of Macedonia



\section{Introduction}

Pulmonary renal syndrome (PRS) is most commonly used to describe a combination of glomerulonephritis and pulmonary hemorrhage as a manifestation of a multisystem autoimmune disease. It is not a single entity but is caused by varied etiologies, including anti-neutrophil cytoplasmic antibody (ANCA), associated small vessel vasculitis (such as Wegener's granulomatosis, microscopic polyangiitis, and Churg-Strauss vasculitis), Goodpasture's syndrome, systemic lupus erythematosus, HenochSchonlein purpura, cryoglobulinemia, and rare causes such as drug-induced vasculitis and subacute endocarditis [1], [2]. However, ANCA-associated vasculitis (AAV) and anti-glomerular basement membrane (anti-GBM) disease account for 70-90\% of these cases [1]. In this life-threatening condition with many non-specific clinical symptoms, it is very important to make early diagnosis and initiate prompt treatment to preserve the organ function. The diagnosis rests on the identification of particular patterns of clinical, radiologic, pathologic, and laboratory features. The majority of cases of PRS are associated with ANCAs, either c-ANCA or p-ANCA, due to autoantibodies against the target antigens proteinase-3 and myeloperoxidase, respectively [3]. The antigen target in Goodpasture's syndrome is type IV collagen, the major component of glomerular and alveolar basement membranes [4]. In over $90 \%$ of the cases, autoantibodies (ANCA and anti-GBM) can be detected, making unclassified PRS a diagnostically challenging entity to the clinician [1]. 
We report a case of a patient who presented with renal and pulmonary manifestations, where the diagnosis was based on clinical manifestations and pathological findings since all commercially available serologic studies were negative.

\section{Case Report}

A 51-year-old man was presented to our hospital approximately 2 months after he had the first symptoms of breathing difficulties, malaise, weight loss, mild hemoptysis, diagnosed corneal ulcer, episodes of febrility, and anemia treated with blood transfusion, but with no evidence of bleeding and already registered deteriorated renal function (creatinine $269 \mu \mathrm{mol} / \mathrm{L}$, blood urea nitrogen $20 \mathrm{mmol} / \mathrm{L}$ ). On admission, his vital signs were as follows: Body temperature $36^{\circ} \mathrm{C}$, heart rate 94 beats/min, peripheral capillary oxygen saturation $\left(\mathrm{SpO}_{2}\right)$ $96 \%$, and blood pressure $135 / 75 \mathrm{mmHg}$. The laboratory results showed leukocytosis (white blood cell count: 13.3), normocytic normochromic anemia (hemoglobin $8.0 \mathrm{~g} / \mathrm{dL}$ ), elevated C-reactive protein level (163 mg/dL), and renal dysfunction (creatinine $525 \mu \mathrm{mol} / \mathrm{L}$, blood urea nitrogen $20 \mathrm{mmol} / \mathrm{L}$ ). Urinalysis showed hematuria and proteinuria $(+++)$. Electrocardiogram showed normal sinus rhythm. Abdominal ultrasound showed bilateral enlarged kidney (left $128 \mathrm{~mm} \times 65 \mathrm{~mm}$, right $138 \mathrm{~mm} \times 69 \mathrm{~mm}$, parenchyma around $19 \mathrm{~mm}$ ) with increased echogenicity. Echocardiography revealed a normal ejection fraction of $60 \%$, normal right ventricular size, severe hypertrophy of interventricular septum, and no evidence of pericardial effusion. Chest X-ray and computed tomography (CT) on admission showed bilateral central opacities, which were more confluent in the right upper and lower lung zone than elsewhere (Figure 1). Gas analysis showed metabolic acidosis compensated by respiratory alkalosis. A preliminary diagnosis of pneumonia was suspected on admission and a combination of several antibiotics was started, but all cultures, including blood and urine remained negative (testing for hepatitis $B$ and $C$ virus, leishmania, pneumoslide, anti-toxoplasma $\lg M$ and $\lg G$, BAB test, and Löwenstein-Jensen). We detected only serological positive test for cytomegalovirus which was treated with valganciclovir of $450 \mathrm{mg}$.

Serological testing for Goodpasture's syndrome (anti-GBM antibodies were performed using enzymelinked immunosorbent assay/ELISA). Vasculitis (proteinase 3/PR3-ANCA/and myeloperoxidase/MPOANCA/) showed negative results, and ELISA was performed twice during the clinical course. We also performed serological tests about anti-phospholipid antibodies, lupus cells, CYFRA, and all were negative (Table 1). Serum complement levels, C3 and C4, were within reference values.
Table 1: Repetitive serological testing

\begin{tabular}{lll}
\hline Serological testing & First measurement & Second measurement \\
\hline $\begin{array}{l}\text { Glomerular basement membrane }(\mathrm{EU} / \mathrm{mL}) \\
\text { negative }<20 \text {; positive }>20\end{array}$ & $<2$ & $<2$ \\
p-ANCA (MPO) negative $<20$; positive $>20$ & 5.74 & 5.52 \\
C-ANCA $(\mathrm{PR} 3)-$ negative $<20$; positive $>20$ & 20.6 & 19.6 \\
Anti-MPO $(\mathrm{U} / \mathrm{mL})-$ negative $<5$ & 1 & 1 \\
Anti-PR3 $(\mathrm{U} / \mathrm{mL})-$ negative $<10$ & 2 & 2 \\
CYFRA $(\mathrm{mg} / \mathrm{ml})-0.10-3.3$ & 5.47 & 1 \\
\hline
\end{tabular}

Kidney biopsy was performed on the $3^{\text {rd }}$ day, and the result of immunofluorescence (IF) was delivered the same day, while the histopathological finding after 10 days (Figure 3a, Figure 3b). Despite negative test results for anti-GBM and ANCA, considering the IF renal biopsy result, we initiated treatment with cyclophosphamide and corticosteroids. Therapy with methylprednisolone $(500 \mathrm{mg} / \mathrm{d})$ was started on day 3 , continued for 3 days, and was followed by therapy with prednisolone $60 \mathrm{mg} /$ day over the next 2 weeks, with a gradual dose reduction in line with KDIGO Clinical Practice Guideline for Glomerulonephritis [5]. Plasma exchange was started on the $4^{\text {th }}$ day and continued in the next 5 days. Cyclophosphamide of $500 \mathrm{mg}$ was also given every 2 weeks for three pulses. The repeated thoracic CT scan showed areas of consolidation with parenchymal ground-glass opacities and a few pulmonary nodules changes (Figure $2 b$ ). On the $37^{\text {th }}$ day, we performed bronchoscopy with biopsy, which revealed macroscopically neat tracheobronchial mucosa, with no fresh bleeding, while the biopsy showed only hemorrhage with fibrosis. Under immunosuppressive and antibiotic therapy, plasma exchange, the patient's status continually improved; there was no pulmonary bleeding, but, unfortunately, serum creatinine level remained high and renal function remained in stage 4 chronic kidney disease, with diuresis to $3000 \mathrm{ml}$. Due to the absence of positive serological antibodies, 24 urinary protein was performed several times and used as a marker to monitor the effect of treatment (Table 2.), but it remained in nephrotic values.

Table 2: Laboratory examination during the first hospitalization

\begin{tabular}{|c|c|c|c|c|c|c|c|c|c|}
\hline Day & 1 & 3 & 7 & 15 & 20 & 25 & 29 & 34 & 38 \\
\hline Hemoglobin $(\mathrm{g} / \mathrm{dL})$ & 8 & 7.6 & 8 & 8.3 & 10.7 & 9.9 & 9.6 & 10.3 & 9.6 \\
\hline Leukocyte $\left(10^{3} / \mathrm{uL}\right)$ & 8 & 13 & 8.8 & 13.9 & 21 & 12.9 & 13.1 & 12.7 & 11.6 \\
\hline Albumin $(\mathrm{g} / \mathrm{L})$ & 32 & 32 & 33 & 32 & 33 & 33 & 30 & 33 & 30 \\
\hline Creatinine (umol/L) & 510 & 525 & 391 & 361 & 226 & 257 & 298 & 323 & 332 \\
\hline $\begin{array}{l}24 \text { urinary } \\
\text { protein-g/day }\end{array}$ & 1 & 2.3 & I & 8.5 & 7 & 1 & 8.1 & 4.6 & 6.8 \\
\hline Diuresis (mL) & 2700 & 4000 & 3300 & 3000 & 4000 & 3700 & 2600 & 3100 & 3000 \\
\hline $\begin{array}{l}\text { C-reactive protein } \\
(\mathrm{mg} / \mathrm{L})\end{array}$ & 163 & 250 & 100 & 32 & 40.2 & 66.9 & 70 & 82 & 60 \\
\hline
\end{tabular}

The patient was discharged 40 days after admission with a maintenance dosage of prednisolone of $30 \mathrm{~g} /$ day, with a recommendation to continue rehabilitation and to return to our hospital after 10 days for the third dose of cyclophosphamide. However, 2 weeks later, due to worsening of his general condition, he was hospitalized again, with symptoms of breathing difficulties, malaise, and hemoptysis. In spite of the CT finding of bilateral bronchopneumonia, we suspected relapse of the pulmonary-renal syndrome. Furthermore, all cultures, including blood and urine remained negative. Serological test for Cytomegalovirus was already negative. Despite repeatedly negative results in laboratory testing for 
anti-GBM and ANCA, therapy with methylprednisolone (250 mg) was started, continued for 3 days (125 mg), and followed by therapy with prednisolone of $30 \mathrm{mg} / \mathrm{day}$. On the $2^{\text {nd }}$ day, due to fluid overload, emergent hemodialysis was done beside impaired renal function. In addition, plasma exchange was started and the patient underwent a total of nine sessions. Due to persistent leukopenia, after the third dose of cyclophosphamide, it was discontinued and immunosuppressive therapy was continued with mycophenolate mofetil of $1 \mathrm{~g} /$ day. Unfortunately, despite diuresis recovery, the attempt to discontinue renal replacement therapy was unsuccessful, and the patient remained dialysis-dependent. He was discharged from the hospital 30 days after admission in a stable general condition, with a maintenance dosage of prednisolone of $30 \mathrm{~g} /$ day, with a recommendation for dose reduction, and mycophenolate mofetil of $1 \mathrm{~g} /$ day, and hemodialysis 3 times/week (Table 3).

Table 3: Laboratory examination during the second hospitalization

\begin{tabular}{llllllll}
\hline Day & 1 & 3 & 8 & 14 & 20 & 27 & 30 \\
\hline Hemoglobin $(\mathrm{g} / \mathrm{dL})$ & 7 & 5.8 & 8.1 & 10.6 & 9.1 & 10.5 & 9.7 \\
Leukocyte $\left(10^{3} / \mathrm{uL}\right)$ & 11.7 & 7.7 & 8.6 & 7.7 & 2.9 & 4.6 & 5.4 \\
Albumin $(\mathrm{g} / \mathrm{L})$ & 33 & 30 & 35 & 40 & & 38 & \\
Creatinine (umol/L) & 558 & 585 & 435 & 402 & & 394 & 336 \\
24 urinary protein-g/day & $I$ & 5.7 & $/$ & 8.1 & $/$ & 5.1 & $/$ \\
Diuresis $(\mathrm{mL})$ & 1440 & 1700 & 2800 & 2200 & 1000 & 1100 & 700 \\
C-reactive protein (mg/L) & 34.7 & 20 & 15 & 10 & 9.4 & 1.6 & 0.7 \\
\hline
\end{tabular}

The maintenance therapy was discontinued after 12 months, and after 24 months, we have received information from the hemodialysis center that he is on regular hemodialysis, in good condition, without pulmonary problems

\section{Discussion}

The term PRS is an extensive term which includes a combination of diffuses pulmonary hemorrhage and glomerulonephritis, which may be a consequence of a multisystem autoimmune disease [1], [2]. Several types of immunologic and non-immunologic mechanisms are involved in the PRS pathogenesis, but the most common are AAV [3] and anti-GBM antibody disease [4]. Given that a significant number of patients present with rapid clinical deterioration, early and accurate diagnosis is essential to apply adequate therapy, which is basically an aggressive one [6]. The gold standard diagnostic test is renal or lung biopsy, along with serological analysis for detection of antibodies, especially ANCA and anti-GBM, which are present in the serum of $90 \%$ of patients with PRS. However, clinicians face major diagnostic and therapeutic challenges in a small group of patients who remain seronegative during the illness. Studies suggest that seronegative anti-GBM disease is very rare, occurring in $2-3 \%$ of cases, while a subgroup ( $10 \%)$ of patients with clinical features and pathology consistent with AAV remains ANCA negative on testing [3]. On the other hand, the concurrence of both ANCA and anti-GBM antibodies in individual patients has been observed in several series [7], but double seronegative is extremely rare. In our double seronegative (ANCA and anti-GBM antibodies) case with PRS, two differential diagnoses for the underlying clinical condition seemed possible: Anti-GBM disease as well as AAV, which opened many diagnostic and therapeutic dilemmas.

In terms of renal biopsy, it is known that IF helps to distinguish between anti-GBM disease and AAV. The typical description of IF findings in anti-GBM disease is the linear deposition of IgG along GBMs [8], while in ANCA-associated glomerulonephritis is the little or no glomerular staining for Igs or complement, the so-called pauci-immune staining pattern [9]. The renal biopsy of our patient showed, on the one hand, deposition of $\lg G$ along GBMs, although not linear, while on the other, crescents at varying stages with extracapillary glomerulonephritis, emphasizing the
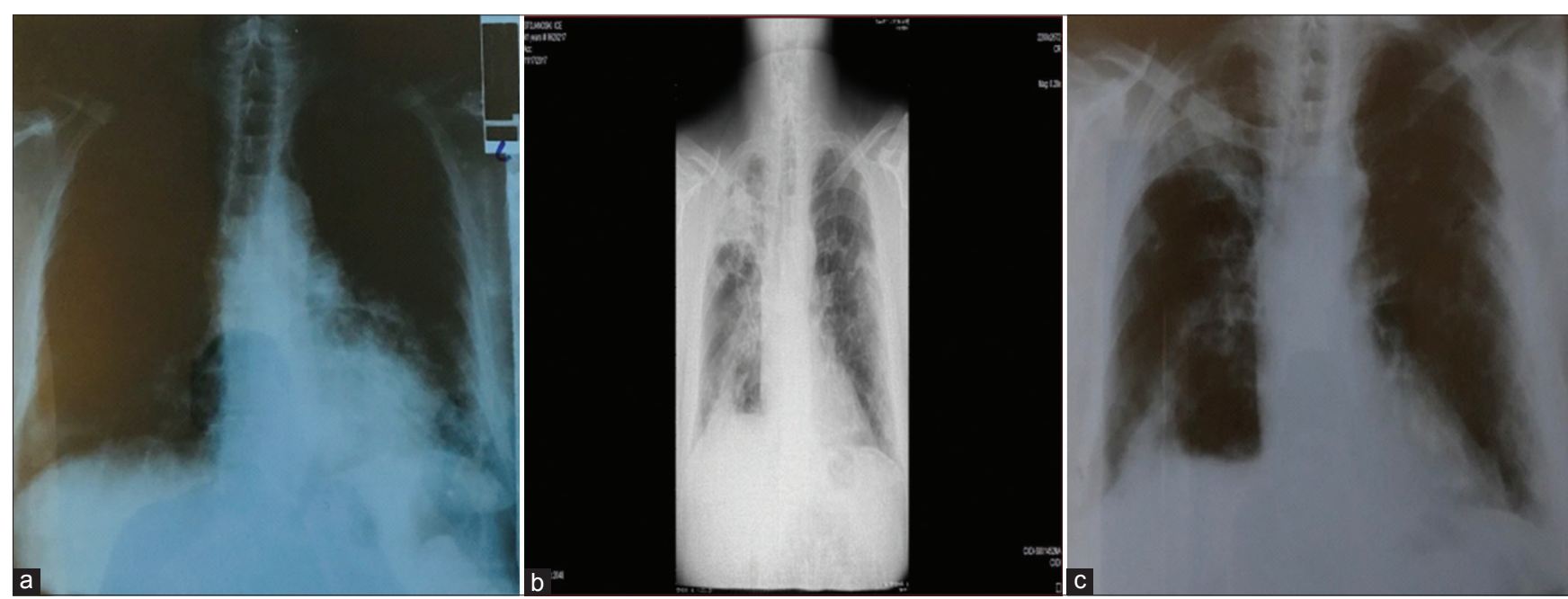

Figure 1: Chest X-ray on admission (a) and during hospitalization ( $b$ and c) - Chest X-ray on admission showed (a) multifocal bronchopneumonia, with bilateral central opacities, which were more confluent in the right upper and lower lung zone than elsewhere; on the $10^{\text {th }}$ and $15^{\text {th }}$ day $(b$ and c) consolidation of the right lung, but with fibrotic changes 


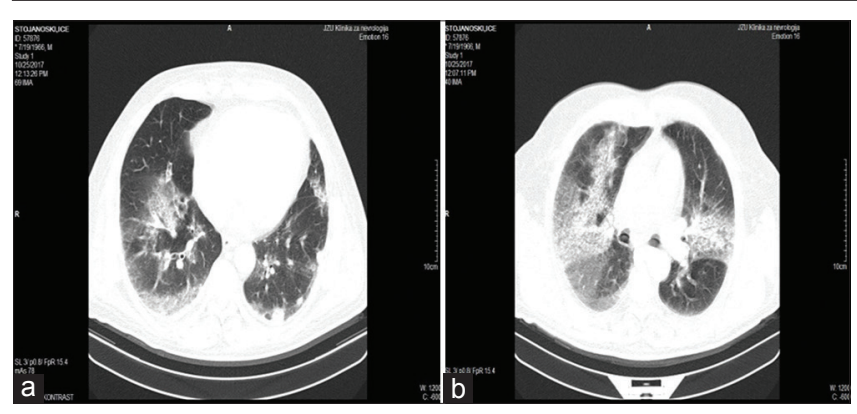

Figure 2: Chest computed tomography (CT) scan on the $8^{\text {th }}$ day showed (a) areas of consolidation with parenchymal ground-glass opacities and a few pulmonary nodules changes. Chest CT scan on the $30^{\text {th }}$ day showed (b) ground-glass opacities, consolidated opacities, and pleural effusions

possibility of a double seronegative PRS with rapidly progressive glomerulonephritis.

In terms of pulmonary involvement in PRS, diffuse alveolar hemorrhage (DAH) represents a broad clinical spectrum. Hemoptysis is the most common but not specific clinical manifestation of DAH [10] was present in our case. The CT of pulmonary involvement in AAV was manifested with multiple nodules of variable size randomly distributed throughout the lungs, with airspace consolidation and ground-glass opacities, while in anti-GBM antibody disease was ground-glass and consolidated opacities that progress to a reticular "crazy paving" pattern over a few weeks [11]. In our case, the presence of nodules was more indicative of $\mathrm{AAV}$, but their non-cavitation and radiographic resolution during follow-up were more in the direction of antiGBM antibody disease. However, once a diagnosis of $\mathrm{DAH}$ is established, the clinician should determine if the underlying cause is present. For this purpose, a bronchoscopy was performed to confirm our suspicion of the chest CT findings and the symptoms of the patient, but the biopsy showed the only hemorrhage with fibrosis.

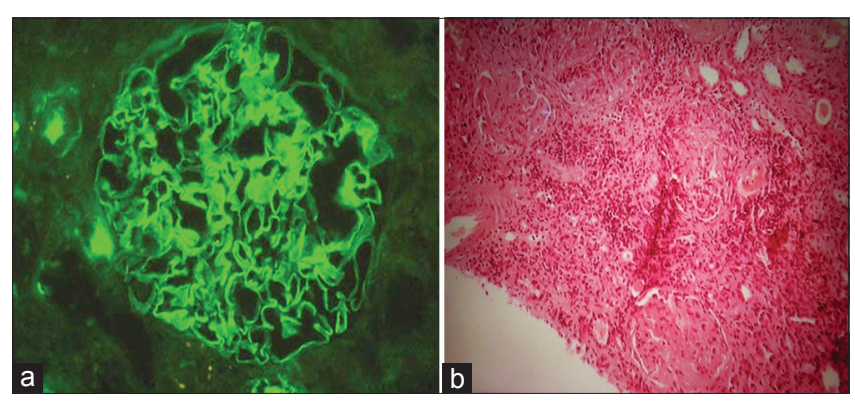

Figure 3: (a) Immunofluorescence was performed on 3 glomeruli, they were all wrapped with cellular crescents at varying stages. IgG1/3 glomeruli showed intensive deposition of IgG in the glomerular basement membrane (+2), but in the glomerulus, the deposition was focal segmental, with present anti-C3 mesangial deposits (b) light microscopy examination performed on 15 glomeruli showed disrupted glomeruli due to pronounced extracapillary cell proliferation and collapsed glomerular basal membrane with expressed fibrin exudation. Tubulointerstitium was with impaired construction due to acute tubular lesion. The finding corresponds to extracapillary glomerulonephritis

In patients with PRS, proteinuria is always present, but rarely in the range of nephrotic syndrome [1].
However, in our case, higher levels of proteinuria were present both on admission and during the treatment. Chen et al. [12] found that ANCA-negative Chinese patients had higher levels of proteinuria than ANCA-positive patients. Furthermore, Adapa et al. found that seronegative atypical anti-GBM crescentic glomerulonephritis was characterized by high-grade proteinuria [13]. In our double seronegative patient, the presence of high proteinuria is another parameter that indicates that two different clinical conditions might overlap. However, although our patient was negative for ANCAs and antiGBM antibodies during hospitalization, Salama et al. reported that it is possible to prove the existence of a low titer antibody against GBM using a biosensor analysis for a patient with anti-GBM disease [14]. Unfortunately, this testing is not available in our laboratory and our country.

Regarding therapeutic dilemmas, although the accurate diagnosis was difficult, we thought that the clinical condition of this double seronegative (ANCA and anti-GBM antibodies) patient could be explained by the parallel presence of both conditions, based on the histopathological finding. Thus, on the $3^{\text {rd }}$ day of hospitalization, after the kidney biopsy, we initiated immunosuppression with cyclophosphamide and corticosteroids. This therapy is identical for both conditions in seropositive patients [5] because of the limited numbers of seronegative PRS. However, along with immunosuppressive therapy, we also started a plasma exchange. It is well known that the basis for consideration of plasma exchange of PRS is the removal of ANCA and anti-GBM antibodies as well as other inflammatory mediators to promote earlier immune response reversal and to minimize tissue damage [3]. The indication for plasma exchange in our case with negative antibodies was pulmonary hemorrhage. A total of 14 plasmapheresis sessions was performed ( 5 in the initial phase and 9 in the relapse state) and the effect was followed by chest CT findings and clinical status.

New diagnostic dilemma arises due to rapid relapse during immunosuppressive therapy and deterioration of renal function that requires hemodialysis treatment. In terms of relapse, in contrast to most other autoimmune kidney diseases, anti-GBM GN is not characterized by a frequently relapsing course, while in terms ofrenalfunction, patients withANCA-related disease with renal failure recover renal function in more than $70 \%$ of the cases, after appropriate immunosuppression. With regard to further treatment, we have decided that the patient should receive maintenance therapy despite being on hemodialysis treatment, which is not in line with the available recommendations [15]. We considered that in this atypical case, intensive plasma exchange and immunosuppressive therapy for anti-GBM disease and $A A V$ are crucial in the early stage and maintenance therapy is necessary for vasculitis in remission.

This reported case has important clinical implications because pulmonary-renal syndrome with negative ANCAs and anti-GBM antibody is extremely 
rare and no treatment recommendations have been established for such cases [16], [17]. Fröhlich-Gildhoff et al., in their interesting study, propose that patients with both circulating anti-GBM and ANCA antibody have a truly hybrid disease phenotype, requiring aggressive early treatment for anti-GBM disease and careful longterm follow-up for maintenance immunosuppression for AAV [7]. Thus, our case raises the question of whether there is another phenotype in double negative patients that requires further investigation to define the underlying mechanisms for the implementation of appropriate treatment strategies.

\section{Conclusion}

Seronegative pulmonary-renal syndrome with the inability of serologically detecting the antibodies (ANCA and anti-GBM) is a challenging entity to the clinician since early diagnosis may be missed leading to delayed appropriate treatment. We think that double negative patients may be more present than described, requiring further investigations to define the underlying mechanisms of this condition and to define optimal treatment strategies.

\section{Authors' Contributions}

SM, AP, VK, and GS were the physicians who treated the patient in this report. The manuscript was prepared by SM and GS. All authors participated in discussions about the manuscript and approved the final version.

\section{Acknowledgments}

This work was presented at the MIRCIM 2019, $5^{\text {th }}$ McMaster International Review Course of Internal Medicine, Krakow, Poland (https://www.mp.pl/paim/en/ node/15081/pdf).

\section{References}

1. Papiris SA, Manali ED, Kalomenidis I, Kapotsis GE Karakatsani A, Roussos C. Bench-to-bedside review: Pulmonary-renal syndromes-an update for the intensivist. Crit Care. 2007;11(3):213. https://doi.org/10.1186/cc5778

\section{PMid:17493292}

2. Gallagher H, Kwan JT, Jayne DR. Pulmonary renal syndrome: A 4-year, single-center experience. Am J Kidney Dis. 2002;39:427. https://doi.org/10.1053/ajkd.2002.29876 PMid: 11774100

3. Geetha D, Jefferson JA. ANCA-associated vasculitis: Core curriculum 2020. Am J Kidney Dis. 2020;75(1):124-37. https:// doi.org/10.1053/j.ajkd.2019.04.031

PMid:31358311

4. Pusey CD. Anti-glomerular basement membrane disease. Kidney Int. 2003;64(4):1535-50. https://doi. org/10.1046/j.1523-1755.2003.00241.x

5. KDIGO Clinical Practice Guideline for Glomerulonephritis. Chapter 13: Pauci-immune focal and segmental necrotizing glomerulonephritis. Kidney Int Suppl. 2012;2:233-42. https://doi. org/10.1038/kisup.2012.26

6. West SC, Arulkumaran N, Ind PW, Pusey CD. Pulmonaryrenal syndrome: A life threatening but treatable condition. Postgrad. 2013;89(1051):274-83. https://doi.org/10.1136/ postgradmedj-2012-131416

PMid:23349383

7. McAdoo SP, Tanna A, Hrušková Z, Holm L, Weiner M, Arulkumaran $\mathrm{N}$, et al. Patients double-seropositive for ANCA and anti-GBM antibodies have varied renal survival, frequency of relapse, and outcomes compared to single-seropositive patients. Kidney Int. 2017;92(3):693-702. https://doi. org/10.1016/j.kint.2017.03.014

PMid:28506760

8. Fogo AB, Lusco MA, Najafian B, Alpers CE. AJKD atlas of renal pathology: Anti-glomerular basement membrane antibodymediated glomerulonephritis. Am J Kidney Dis. 2016;68(5):e2930. https://doi.org/10.1053/j.ajkd.2016.09.003

9. Fogo AB, Lusco MA, Najafian B, Alpers CE. AJKD atlas of renal pathology: Pauci-immune necrotizing crescentic glomerulonephritis. Am J Kidney Dis. 2016;68(5):31-2. https:// doi.org/10.1053/j.ajkd.2016.09.002

10. Collard HR, Schwarz MI. Diffuse alveolar hemorrhage. Clin Chest Med. 2004;25(3):583-92. https://doi.org/10.1016/j. ccm.2004.04.007 PMid:15331194

11. Li J, Li C, Li J. Thoracic manifestation of Wegener's granulomatosis: Computed tomography findings and analysis of misdiagnosis. Exp Ther Med. 2018;16(1):413-9. https://doi. org/10.3892/etm.2018.6154

PMid:29896268

12. Chen M, Yu F, Wang SX, Zou WZ, Zhao MH, Wang HY Antineutrophil cytoplasmic autoantibody-negative pauciimmune crescentic glomerulonephritis. J Am Soc Nephrol 2007;18(2):599-605. https://doi.org/10.1681/asn.2006091021 PMid: 17215440

13. Adapa S, Madhav Konala V, Hou J, Naramala S, Agrawal N, Dhingra $\mathrm{H}$, et al. Seronegative atypical anti-glomerular basement membrane crescentic glomerulonephritis. Ann Transl Med. 2019;7(11):246. https://doi.org/10.21037/ atm.2019.04.60 PMid:31317016

14. Salama AD, Dougan T, Levy JB, Cook HT, Morgan SH, Naudeer $\mathrm{S}$, et al. Goodpasture's disease in the absence of circulating anti-glomerular basement membrane antibodies as detected by standard techniques. Am J Kidney Dis. 2002;39(6):1162-7. https://doi.org/10.1053/ajkd.2002.33385 PMid:12046026

15. Rovin BH, Caster DJ, Cattran DC, Gibson KL, Hogan JJ, Moeller MJ, et al. Management and treatment of glomerular diseases (part 2): Conclusions from a kidney disease: 
Improving global outcomes (KDIGO) controversies conference. Kidney Int. 2019;95(2):281-95. https://doi. org/10.36485/1561-6274-2020-24-2-22-41

PMid:30665569

16. Yamaguchi H, Shirakami A, Haku T, Taoka T, Nakanishi $Y$, Inai $\mathrm{T}$, et al. Pulmonary-renal syndrome with negative ANCAs and anti-GBM antibody. Case Rep Nephrol. 2013;2013:434531. https://doi.org/10.1155/2013/434531

17. Fröhlich-Gildhoff $M$, Jabs WJ, Berhold C, Kuhlmann MK, Ketterer $\mathrm{U}$, Kische $\mathrm{S}$, et al. A rare clinical course of seronegative pulmonary-renal syndrome. Case Rep Crit Care. 2016;2016:4893496. https://doi.org/10.1155/2016/4893496 